\title{
On the six components of optical angular momentum
}

\author{
Stephen M. Barnett \\ SUPA, Department of Physics, Unniversity of Strathclyde, Glasgow G4 0NG, UK \\ E-mail: steve@phys.strath.ac.uk
}

\begin{abstract}
In special relativity the angular momentum is a rank-two antisymmetric tensor with six independent components. Three of these are the familiar generators of spatial rotation, which for light have been studied at length. The remaining three, which are responsible for the Lorentz boosts, have largely been neglected. We introduce the latter and compare their properties with those of the more familiar generators of rotations. The seemingly natural separation of the generators of Lorentz boosts into spin and orbital parts fails, however, as the spin part is identically zero.
\end{abstract}

Keywords: Optical angular momentum, Lorentz boosts 


\section{Introduction}

Optical angular momentum is rapidly becoming a mature field of research with a number of books and review articles devoted to it $[1,2,3]$. The key idea was the observation that a beam of light prepared in a Laguerre-Gaussian mode carries orbital angular momentum about the beam axis [4]. This is in addition to the more familiar spin angular momentum associated with circular polarization.

The angular momentum carried by a beam of light can exert a torque on a trapped particle causing it to rotate $[5,6]$. The spin and orbital parts of the optical angular momentum cause a small particle to rotate about the centre of the particle or the centre of the beam respectively [7]. In the quantum regime, the production of pairs of photons in spontaneous parametric down-conversion conserves orbital angular momentum leading to entanglement in this quantity [8,9]. Most recently, this has been used to demonstrate, experimentally, an EPR paradox based on the conjugate variables angular momentum and angular position [10, 11].

The separation of optical angular momentum is straightforward only within the paraxial regime and for the components parallel to the beam axis. Once we move beyond this limit the separation into spin and orbital parts becomes more problematic $[12,13,14]$. It is known that a separation into spin and orbital parts is possible beyond the paraxial regime but that neither part alone is itself an angular momentum $[15,16]$. The problem is the difficulty in performing a rotation whilst preserving the transversality of the field. We can, however, identify the spin and orbital parts as the generators of the closest allowed approximation to independent rotation of the directions of the fields (spin part) and the rotation of spatial dependence without changing the directions of the field vectors [17].

In special relativity the angular momentum is an antisymmetric rank-two tensor [18]. This means that it has six independent components. Three of these are the familiar spatial angular momenta that generate spatial rotations. The remaining three are the generators of the Lorentz boosts [19], one for each of the three orthogonal cartesian directions. They may be thought of as the generators of rotations in the sense that they couple a cartesian direction with time. Like the more familiar spatial angular momenta, the generators of boosts, for the free electromagnetic field, are constants of the motion. This is true even though they depend explicitly on time. In this paper we introduce the generators of boosts and compare and contrast their properties with those of the remaining three more familar angular momenta. As a first step in exploring their properties we ask whether a separation into spin and orbital parts may be performed by following those techniques previously applied to the generators of spatial rotations $[17]$. 


\section{Rotations and boosts}

We shall identify our six components of the optical angular momentum with the infinitessimal generators of rotations and Lorentz boosts. As an introduction we first remind the reader about the forms of these transformations. We work throughout with a rationalized system of units in which $\varepsilon_{0}=\mu_{0}=c=1$, so that Maxwell's equations for the free field become

$$
\begin{aligned}
& \boldsymbol{\nabla} \cdot \mathbf{E}=0 \\
& \boldsymbol{\nabla} \cdot \mathbf{B}=0 \\
& \boldsymbol{\nabla} \times \mathbf{E}=-\frac{\partial}{\partial t} \mathbf{B} \\
& \boldsymbol{\nabla} \times \mathbf{B}=\frac{\partial}{\partial t} \mathbf{E} .
\end{aligned}
$$

Let us consider first a rotation of the fields. The transformation that achieves this has to do two things: (i) it must change the spatial coordinates in the arguments of the fields and (ii) it must change the orientations of the fields. For the sake of definiteness, we specify a rotation through angle $\theta$ about the $x$-axis. This means rotating the spatial coordinates in the arguments of the fields in the opposite direction:

$$
\begin{aligned}
& x \rightarrow x^{\prime}=x \\
& y \rightarrow y^{\prime}=\cos \theta y+\sin \theta z \\
& z \rightarrow z^{\prime}=\cos \theta z-\sin \theta y .
\end{aligned}
$$

It is also necessary to rotate the directions of the fields:

$$
\begin{aligned}
& E_{x} \rightarrow E_{x} \\
& E_{y} \rightarrow \cos \theta E_{y}-\sin \theta E_{z} \\
& E_{z} \rightarrow \cos \theta E_{z}+\sin \theta E_{y} .
\end{aligned}
$$

If we combine these two contributions we find

$$
\begin{aligned}
& E_{x}(\mathbf{r}, t) \rightarrow E_{x}^{\prime}\left(\mathbf{r}^{\prime}, t\right)=E_{x}(\mathbf{r}, t) \\
& E_{y}(\mathbf{r}, t) \rightarrow E_{y}^{\prime}\left(\mathbf{r}^{\prime}, t\right)=\cos \theta E_{z}(\mathbf{r}, t)-\sin \theta E_{z}(\mathbf{r}, t) \\
& E_{z}(\mathbf{r}, t) \rightarrow E_{z}^{\prime}\left(\mathbf{r}^{\prime}, t\right)=\cos \theta E_{z}(\mathbf{r}, t)+\sin \theta E_{y}(\mathbf{r}, t) .
\end{aligned}
$$

Our principal interest will be with the infinitessimal transformations that are first order in $\theta$. It is convenient to write these in terms of the orginal unprimed coordinates:

$$
\begin{aligned}
& E_{x}(\mathbf{r}, t) \rightarrow E_{x}(\mathbf{r}, t)-\theta\left(y \frac{\partial}{\partial z}-z \frac{\partial}{\partial y}\right) E_{x}(\mathbf{r}, t) \\
& E_{y}(\mathbf{r}, t) \rightarrow E_{y}(\mathbf{r}, t)-\theta\left(y \frac{\partial}{\partial z}-z \frac{\partial}{\partial y}\right) E_{y}(\mathbf{r}, t)-\theta E_{z}(\mathbf{r}, t) \\
& E_{z}(\mathbf{r}, t) \rightarrow E_{z}(\mathbf{r}, t)-\theta\left(y \frac{\partial}{\partial z}-z \frac{\partial}{\partial y}\right) E_{z}(\mathbf{r}, t)+\theta E_{y}(\mathbf{r}, t) .
\end{aligned}
$$

The magnetic field transforms in the same fashion, with $E$ simply replaced by $B$. 
The Lorentz boosts correspond to changing from one inertial frame to a second moving with velocity $\mathbf{v}$ relative to the first. For the sake of definiteness let us specify that this velocity is in the $x$-direction. As with the rotation, our transformation needs to do two things (i) it must change the sptial and temporal coordinates in the arguments of the fields and (ii) it must mix the electric and magnetic fields. This means changing both the spatial and temporal coordinates in the arguments of the fields by the Lorentz transformation [19]

$$
\begin{aligned}
& x \rightarrow x^{\prime}=\gamma(x+v t) \\
& y \rightarrow y^{\prime} \\
& z \rightarrow z^{\prime} \\
& t \rightarrow t^{\prime}=\gamma(t+v x),
\end{aligned}
$$

where $\gamma=\left(1-v^{2}\right)^{-1 / 2}$. It is also necessary to superpose the electric and magnetic fields from the orginal frame $[20]$ :

$$
\begin{aligned}
& E_{x} \rightarrow E_{x} \\
& E_{y} \rightarrow \gamma\left(E_{y}+v B_{z}\right) \\
& E_{z} \rightarrow \gamma\left(E_{z}-v B_{y}\right) .
\end{aligned}
$$

We can think of this as a rotation between the electric and magnetic fields through an imaginary angle so that the trigonometrical functions in the spatial rotation are replaced by hyperbolic ones with argument $\phi=\tanh ^{-1} v$.

As with rotations, we shall primarily be interested in the infinitessimal transformations which are first order in $v$. As with the rotations, it is convenient to write these in terms of the orginal unprimed coordinates:

$$
\begin{aligned}
& E_{x}(\mathbf{r}, t) \rightarrow E_{x}(\mathbf{r}, t)+v\left(x \frac{\partial}{\partial t}+t \frac{\partial}{\partial x}\right) E_{x}(\mathbf{r}, t) \\
& E_{y}(\mathbf{r}, t) \rightarrow E_{y}(\mathbf{r}, t)+v\left(x \frac{\partial}{\partial t}+t \frac{\partial}{\partial x}\right) E_{y}(\mathbf{r}, t)+v B_{z}(\mathbf{r}, t) \\
& E_{z}(\mathbf{r}, t) \rightarrow E_{z}(\mathbf{r}, t)+v\left(x \frac{\partial}{\partial t}+t \frac{\partial}{\partial x}\right) E_{z}(\mathbf{r}, t)-v B_{y}(\mathbf{r}, t) .
\end{aligned}
$$

The corresponding transformation of the magnetic field is

$$
\begin{aligned}
& B_{x}(\mathbf{r}, t) \rightarrow B_{x}(\mathbf{r}, t)+v\left(x \frac{\partial}{\partial t}+t \frac{\partial}{\partial x}\right) B_{x}(\mathbf{r}, t) \\
& B_{y}(\mathbf{r}, t) \rightarrow B_{y}(\mathbf{r}, t)+v\left(x \frac{\partial}{\partial t}+t \frac{\partial}{\partial x}\right) B_{y}(\mathbf{r}, t)-v E_{z}(\mathbf{r}, t) \\
& B_{z}(\mathbf{r}, t) \rightarrow B_{z}(\mathbf{r}, t)+v\left(x \frac{\partial}{\partial t}+t \frac{\partial}{\partial x}\right) B_{z}(\mathbf{r}, t)+v E_{y}(\mathbf{r}, t) .
\end{aligned}
$$

It is straighforward to show that both sets of transformed fields satisfy Maxwell's equations and, in particular, that they are transverse (divergenceless) fields. 


\section{Generators of rotations and boosts}

In special relativity the angular momentum is a rank-two antisymmetric tensor

$$
L^{\mu \nu}=\int d^{3} r\left(x^{\mu} T^{0 \nu}-x^{\nu} T^{0 \mu}\right),
$$

where $x^{\mu}$ are the four space-time coordinates $(\mu=0,1,2,3)$ and $T^{\mu \nu}$ is the symmetric energy stess tensor [21], the relevant elements of which are

$$
\begin{aligned}
& T^{00}=\frac{1}{2}\left(E^{2}+B^{2}\right) \\
& T^{0 i}=(\mathbf{E} \times \mathbf{B})_{i}=\varepsilon_{i j k} E_{j} B_{k} .
\end{aligned}
$$

Here we have introduced the fully antisymmetric alternating symbol $\varepsilon_{i j k}$ and also the summation convention in which there is an implied summation over indices appearing precisely twice in any given term [22]. The three fully spatial components of the angular momentum tensor (10) are

$$
L^{i j}=\int d^{3} r\left[r_{i}(\mathbf{E} \times \mathbf{B})_{j}-r_{j}(\mathbf{E} \times \mathbf{B})_{i}\right] .
$$

We can clearly identify this with the $k$ component of angular momentum, $J_{k}=\frac{1}{2} \varepsilon_{i j k} L^{i j}$ or, in more familiar form,

$$
\mathbf{J}=\int d^{3} r \mathbf{r} \times(\mathbf{E} \times \mathbf{B}) .
$$

This angular momentum is a constant of the motion in that

$$
\frac{d \mathbf{J}}{d t}=0 \text {. }
$$

This conservation law is a consequence, of course, of the isotropy of free space. The angular momentum is also the generator of infinitessimal rotations of the electric and magnetic fields. It generates the transformations [17] $\ddagger$

$$
\begin{aligned}
& \mathbf{E} \rightarrow \mathbf{E}-[\boldsymbol{\theta} \cdot(\mathbf{r} \times \boldsymbol{\nabla})] \mathbf{E}+\boldsymbol{\theta} \times \mathbf{E} \\
& \mathbf{B} \rightarrow \mathbf{B}-[\boldsymbol{\theta} \cdot(\mathbf{r} \times \boldsymbol{\nabla})] \mathbf{B}+\boldsymbol{\theta} \times \mathbf{B},
\end{aligned}
$$

where the direction of $\boldsymbol{\theta}$ is the axis of the rotation and its magnitude (which is small) is the angle of rotation. These are simply the infinitessimal rotations (5) written in a more condensed notation.

The remaining three components mix space and time. We denote these by $K_{i}$ so that

$$
K_{i}=L^{i 0}=\int d^{3} r\left[\frac{r_{i}}{2}\left(E^{2}+B^{2}\right)-t \varepsilon_{i j k} E_{j} B_{k}\right]
$$

or, more simply,

$$
\mathbf{K}=\int d^{3} r\left[\frac{\mathbf{r}}{2}\left(E^{2}+B^{2}\right)-t \mathbf{E} \times \mathbf{B}\right]
$$

$\ddagger$ Note that here we are considering active rotations rather than the passive rotations discussed in [17] and this accounts for the diffrent signs. 
In the service of clarity, we shall refer to this quantity as the boost angular momentum and to $\mathbf{J}$ as the rotation angular momentum. Like the more familiar rotation angular momentum the boost angular momentum is a constant of the motion in that

$$
\frac{d \mathbf{K}}{d t}=0 \text {. }
$$

The proof of this follows from application of Poynting's theorem [21] and corresponds to Newton's first law of motion as generalised to apply to the centre of energy [18]. A short outline of this is given in Appendix A. The components of the boost angular momentum are the generators of infinitessimal generators of Lorentz transformations:

$$
\begin{aligned}
& \mathbf{E} \rightarrow \mathbf{E}+\left[\boldsymbol{\phi} \cdot\left(\mathbf{r} \frac{\partial}{\partial t}+t \boldsymbol{\nabla}\right)\right] \mathbf{E}-\boldsymbol{\phi} \times \mathbf{B} \\
& \mathbf{B} \rightarrow \mathbf{B}+\left[\boldsymbol{\phi} \cdot\left(\mathbf{r} \frac{\partial}{\partial t}+t \boldsymbol{\nabla}\right)\right] \mathbf{B}+\boldsymbol{\phi} \times \mathbf{E} .
\end{aligned}
$$

Here the direction of $\phi$ is the direction of the relative motion of the new frame and its magnitude is the (small) relative speed $\phi=\tanh ^{-1} v \approx v$. A brief derivation of this important result is given in Appendix B. We recognise these as the infinitessimal boost, given in (8) and (9), written in a more condensed notation. We can iterate this infinitessimal transformation to obtain the familiar transformations of the fields between frames differing by a macroscopic velocity [20, 23].

It is worth pausing to comment on the points of similarity and difference between the two sets of transformations (15) and (19). We recall that each transformation is required to do two things: (i) to change the spatial and temporal coordinates in the arguments of the fields and (ii) to change the orientations of the fields, for the rotation, or to mix the electric and magnetic fields, for the boost. The two terms in the changes of the fields (15) and (19) correspond to these two changes. We should emphasise that only the combination of both changes corresponds to a physical transformation of the fields.

Let us consider first the changes of the coordinates. The operator $\boldsymbol{\theta} \cdot(\mathbf{r} \times \boldsymbol{\nabla})$ generates a rotation in the spatial coordinates about the $\boldsymbol{\theta}$ axis:

$$
\mathbf{r}^{\prime}=[1+\boldsymbol{\theta} \cdot(\mathbf{r} \times \boldsymbol{\nabla})] \mathbf{r}=\mathbf{r}+\boldsymbol{\theta} \times \mathbf{r},
$$

which is the infinitessimal form of the rotation of $\mathbf{r}$. This transformation, being a spatial rotation, leaves the temporal coordinate unchanged. The operator $\phi \cdot\left(\mathbf{r} \frac{\partial}{\partial t}+t \boldsymbol{\nabla}\right)$ generates something akin to a rotation in space-time to change both the spatial and temporal coordinates:

$$
\begin{aligned}
& \mathbf{r}^{\prime}=\left[1-\boldsymbol{\phi} \cdot\left(\mathbf{r} \frac{\partial}{\partial t}+t \boldsymbol{\nabla}\right)\right] \mathbf{r}=\mathbf{r}-\boldsymbol{\phi} t \\
& t^{\prime}=\left[1-\boldsymbol{\phi} \cdot\left(\mathbf{r} \frac{\partial}{\partial t}+t \boldsymbol{\nabla}\right)\right] t=t-\boldsymbol{\phi} \cdot \mathbf{r}
\end{aligned}
$$

which we recognise as the infinitessimal form of the Lorentz boost associated with moving to a frame with velocity $\phi$. When combined and multiplied by $i$, the six operators 
$\mathbf{r} \times \nabla$ and $\mathbf{r} \frac{\partial}{\partial t}+t \boldsymbol{\nabla}$ form a set of six generalized angular momentum operators and allow us to generate any transformation in the Lorentz group [24].

The second parts of our transformations are the changes in direction of the fields or the mixing of the electric and magnetic fields. For the rotation angular momentum the electic and magnetic field are rotated about the $\boldsymbol{\theta}$ axis by the infinitessimal angle $\theta$ :

$$
\begin{aligned}
& \mathbf{E} \rightarrow \mathbf{E}+\boldsymbol{\theta} \times \mathbf{E} \\
& \mathbf{B} \rightarrow \mathbf{B}+\boldsymbol{\theta} \times \mathbf{B} .
\end{aligned}
$$

For the boost angular momentum the corresponding transformations are

$$
\begin{aligned}
& \mathrm{E} \rightarrow \mathrm{E}-\phi \times \mathrm{B} \\
& \mathrm{B} \rightarrow \mathrm{B}+\phi \times \mathbf{E},
\end{aligned}
$$

which we recognise as the form of the Lorentz transformation, as valid for an infinitessimal velocity $\phi$. It may not be obvious that we should associate the Lorentz boost with a rotation. To better understand why this is the case, it is helpful to combine the electric and magnetic fields in the Riemann-Silberstein complex vector field $[25,26,27,28,29]$

$$
\mathbf{F}=\frac{1}{\sqrt{2}}(\mathbf{E}+i \mathbf{B}) \text {. }
$$

If we then write the transformations (22) and (23) in terms of the Riemann-Silberstein vector we find

$$
\begin{aligned}
& \mathbf{F} \rightarrow \mathbf{F}+\boldsymbol{\theta} \times \mathbf{F} \\
& \mathbf{F} \rightarrow \mathbf{F}+i \boldsymbol{\phi} \times \mathbf{F},
\end{aligned}
$$

respectively. Hence we can think of the Lorentz boost as a rotation through an imaginary angle. The presence of the plus sign in the operator $\mathbf{r} \frac{\partial}{\partial t}+t \boldsymbol{\nabla}$, as opposed to the more familiar minus sign implicit in $\mathbf{r} \times \boldsymbol{\nabla}$ may also be traced back to the idea of a rotation through an imaginary angle.

\section{The Heaviside-Larmor symmetry}

As a necessary preliminary to exploring the spin and orbital parts of the angular momentum we first describe a symmetry of the free-field Maxwell equations (1), due to Heaviside and Larmor [30, 31], that the equations are unchanged on interchanging the electric and magnetic fields $(\mathbf{E} \rightarrow \mathbf{B}, \mathbf{B} \rightarrow-\mathbf{E})$. More generally they are unchanged by the duplex transformation [21]

$$
\begin{aligned}
& \mathbf{E} \rightarrow \cos \psi \mathbf{E}+\sin \psi \mathbf{B} \\
& \mathbf{B} \rightarrow \cos \psi \mathbf{B}-\sin \psi \mathbf{E},
\end{aligned}
$$

for any angle $\psi$. All physical properties of the field are required to be invariant under this transformation [32], a principle recently described as electric-magnetic democracy [33]. This requirement is equivalent to the statement that all physical quantities can 
depend on the magnitude and direction of the Riemann-Silberstein vector but not on its phase [27]. It is clear that the energy density, $\frac{1}{2}\left(E^{2}+B^{2}\right)$, and the momentum density, $\mathbf{E} \times \mathbf{B}$, both satisfy this requirement. It follows, therefore, that our six components of the optical angular momentum, $\mathbf{J}$ and $\mathbf{K}$, also satisfy this requirement.

The separation of the optical angular momentum into spin and orbital requires us to introduce the vector potential $\mathbf{A}$ [34]. In order that we can satisfy explicitly the Heaviside-Larmor symmetry we also introduce a second vector potential $\mathbf{C}$ [28]. We choose both potentials to be transverse fields $(\boldsymbol{\nabla} \cdot \mathbf{A}=0=\boldsymbol{\nabla} \cdot \mathbf{C})$ and can then write the electric and magnetic fields in terms of either potential:

$$
\begin{aligned}
& \mathbf{E}=-\frac{\partial}{\partial t} \mathbf{A}=-\boldsymbol{\nabla} \times \mathbf{C} \\
& \mathbf{B}=\boldsymbol{\nabla} \times \mathbf{A}=-\frac{\partial}{\partial t} \mathbf{C} .
\end{aligned}
$$

In order to preserve the Heaviside-Larmor symmetry we require that any physical quantity, when expressed in terms of the potentials, be invariant under the transformation [17]

$$
\begin{aligned}
& \mathbf{A} \rightarrow \cos \psi \mathbf{A}+\sin \psi \mathbf{C} \\
& \mathbf{C} \rightarrow \cos \psi \mathbf{C}-\sin \psi \mathbf{A} .
\end{aligned}
$$

Taking the curl or the time derivative of these gives the duplex transformation (26).

\section{Spin and orbital parts}

It has long been understood that it is possible to split the total optical rotation angular momentum into spin and orbital components as follows [34]:

$$
\begin{aligned}
\mathbf{J} & =\int d^{3} r \mathbf{r} \times(\mathbf{E} \times \mathbf{B}) \\
& =\int d^{3} r\left[E_{i}(\mathbf{r} \times \nabla) A_{i}+\mathbf{E} \times \mathbf{A}\right],
\end{aligned}
$$

where we have made use of Gauss's theorem and assumed that the fields fall off to zero sufficiently quickly at large distances to make the resulting surface term equal to zero. It is then natural to consider the first term to be the orbital component and the second the spin part, although neither of these alone is a true angular momentum $[15,16]$.

It has been argued that the spin and orbital parts in (29) might not be acceptable because they do not explicitly satisfy the Heaviside-Larmor symmetry [35]. We can test this idea by writing the angular momentum in a form that explicitly satisfies this symmetry [17]

$$
\mathbf{J}=\frac{1}{2} \int d^{3} r\left[E_{i}(\mathbf{r} \times \nabla) A_{i}+B_{i}(\mathbf{r} \times \nabla) C_{i}+\mathbf{E} \times \mathbf{A}+\mathbf{B} \times \mathbf{C}\right] .
$$

It is straightforward to show, using appropriate identities from vector calculus and the transversality of $\mathbf{A}$ and $\mathbf{B}$, that this is equal to (29) and, in particular, that the total spin part of the rotation angular momentum is

$$
\mathbf{S}=\int d^{3} r \mathbf{E} \times \mathbf{A}=\frac{1}{2} \int d^{3} r[\mathbf{E} \times \mathbf{A}+\mathbf{B} \times \mathbf{C}],
$$


which clearly satisfies the Heaviside-Larmor symmetry. It should be noted, however, that we can rule out the quantity $\mathbf{E} \times \mathbf{A}$ as a candidate for the density of the spin angular momentum as this does not respect the Heaviside-Larmor symmetry. The quantity $\frac{1}{2}(\mathbf{E} \times \mathbf{A}+\mathbf{B} \times \mathbf{C})$, however, does satisfy the symmetry requirement [17].

It is natural to ask whether a similar separation, into spin and orbital parts, is possible for the three components of the boost optical angular momentum. In order to explore this possibility we use the curl identities (27) to write

$$
\begin{aligned}
\mathbf{K}=\int d^{3} r\left\{\frac{\mathbf{r}}{2}\right. & {[-\mathbf{E} \cdot(\boldsymbol{\nabla} \times \mathbf{C})+\mathbf{B} \cdot(\boldsymbol{\nabla} \times \mathbf{A})] } \\
& \left.-\frac{t}{2}[\mathbf{E} \times(\boldsymbol{\nabla} \times \mathbf{A})-\mathbf{B} \times(\boldsymbol{\nabla} \times \mathbf{C})]\right\} .
\end{aligned}
$$

Integrating by parts, assuming that the fields fall off sufficiently quickly at infinity and using Maxwell's equations then gives

$$
\begin{gathered}
\mathbf{K}=\frac{1}{2} \int d^{3} r\left[A_{j}\left(\mathbf{r} \frac{\partial}{\partial t}+t \boldsymbol{\nabla}\right) E_{j}+C_{j}\left(\mathbf{r} \frac{\partial}{\partial t}+t \boldsymbol{\nabla}\right) B_{j}\right] \\
+\frac{1}{2} \int d^{3} r[\mathbf{C} \times \mathbf{E}-\mathbf{A} \times \mathbf{B}] .
\end{gathered}
$$

We recognise the combinations $\mathbf{r} \frac{\partial}{\partial t}+t \boldsymbol{\nabla}$ from our (infinitessimal) Lorentz transformations (19) and it is tempting, therefore, to associate the first integral with the orbital part of $\mathbf{K}$ and the second with the spin part. There is a problem with this line of reasoning, however. To see this we evaluate the integral of the $\mathbf{A} \times \mathbf{B}$ term:

$$
\begin{aligned}
-\int d^{3} r(\mathbf{A} \times \mathbf{B})_{i} & =-\int d^{3} r[\mathbf{A} \times(\boldsymbol{\nabla} \times \mathbf{A})]_{i} \\
& =-\int d^{3} r A_{j} \nabla_{i} A_{j}-A_{j} \nabla_{j} A_{i} \\
& =-\int d^{3} r \nabla_{j}\left(\frac{1}{2} \delta_{i j} A^{2}-A_{j} A_{i}\right) \\
& =-\oint\left(\frac{1}{2} \delta_{i j} A^{2}-A_{j} A_{i}\right) d s_{j} \\
& =0,
\end{aligned}
$$

where we have use the transversality of $\mathbf{A}$, Gauss's theorem and assumed that the fields fall to zero sufficiently quickly at infinity. A similar calculation reveals that the integral of $\mathbf{C} \times \mathbf{E}$ is also zero and hence the integral in the second line of (33) is identically zero! The first line of (33) therefore comprises the totality of our boost optical angular momentum. It seems that for these three components there is no simple separation into spin and orbital components to match that of the more familiar rotation angular momentum. It remains unclear whether or not the non-zero quantity $\frac{1}{2}(\mathbf{C} \times \mathbf{E}-\mathbf{A} \times \mathbf{B})$ may have a physical significance as a local density.

\section{Conclusion}

When viewed from the perspective of special relativity, the angular momentum is naturally a six-component quantity. Three of these, the components of the rotation 
angular momentum, are the familiar generators of rotations about an axis in space. The remaining three, the components of the boost angular momentum, generate the Lorentz boosts between different inertial frames. These boosts may be understood as rotations by an imaginary angle, coupling the spatial coordinates with time.

The familiar fully spatial components of the rotation angular momentum may be split into spin and orbital parts in a natural way, although neither of these component parts is itself a true angular momentum $[15,16]$. We have seen how the HeavisideLarmor, or duplex, symmetry suggests a more explicitly symmetric form for these quantities [17] and argued that it is only such a symmetric quantity that can be a candidate for a local density of spin or orbital angular momentum.

The introduction of the second vector potential $\mathbf{C}$ [28] enabled us to carry out a splitting of the boost angular momentum in a manner analogous to that for the rotation angular momentum. We found, however, that the resulting spin-like component of the boost angular momentum is identically zero. It may be possible to attribute some physical significance to spin and orbital densities of boost angular momentum but not, it seems, to separate total spin and orbital components of the boost angular momentum.

\section{Acknowledgments}

I thank Les Allen, Alison Yao and Miles Padgett for helpful comments and suggestions. I am also grateful to an anonymous referee whose comments both corrected a number of errors and, I hope, greatly clarified the presentation. This work was supported by the UK Engineering and Physical Sciences Research Council, by the Royal Society and by the Wolfson Foundation.

\section{Appendix A. Conservation of $\mathrm{K}$}

To prove the conservation of $\mathbf{K}$ we evaluate its time derivative to give

$$
\frac{d \mathbf{K}}{d t}=\int d^{3} r \frac{\mathbf{r}}{2} \frac{d}{d t}\left(E^{2}+B^{2}\right)-\int d^{3} r \mathbf{E} \times \mathbf{B}-t \frac{d}{d t} \int d^{3} r \mathbf{E} \times \mathbf{B} .
$$

The final integral is zero by virtue of the global conservation of linear momentum. Poynting's theorem expresses the local conservation of energy in the form

$$
\frac{d}{d t} \frac{1}{2}\left(E^{2}+B^{2}\right)+\nabla \cdot(\mathbf{E} \times \mathbf{B})=0 .
$$

This allows us to rewite (A.1) as

$$
\begin{aligned}
\frac{d \mathbf{K}}{d t} & =-\int d^{3} r \mathbf{r} \boldsymbol{\nabla} \cdot(\mathbf{E} \times \mathbf{B})-\int d^{3} r \mathbf{E} \times \mathbf{B} \\
& =\int d^{3} r[(\mathbf{E} \times \mathbf{B}) \cdot \nabla] \mathbf{r}-\int d^{3} r \mathbf{E} \times \mathbf{B} \\
& =0,
\end{aligned}
$$

where we have used integration by parts to obtain the second line. 


\section{Appendix B. Generation of Lorentz boosts}

We have identified the quantity $\mathbf{K}$ with the Lorentz boosts of the electric and magnetic fields. We can demonstrate this directly by calculating the infinitessimal transformations generated by the action of $\mathbf{K}$. We can perform the transformation within the framework of either classical or quantum electrodynamics but, for definiteness, we choose the quantum description which requires us to introduce the equal-time commutation relation

$$
\left[E_{i}(\mathbf{r}), B_{j}\left(\mathbf{r}^{\prime}\right)\right]=i \hbar \varepsilon_{i j k} \nabla_{k}^{\prime} \delta\left(\mathbf{r}-\mathbf{r}^{\prime}\right) .
$$

The operators $\mathbf{K}$ may be used to generate a unitary transformation of the electric and magnetic fields. It suffices for our purposes to consider only infinitessimal transformations (with $\phi$ small) for which the electric field becomes

$$
\begin{aligned}
\exp \left(\frac{i}{\hbar} \boldsymbol{\phi} \cdot \mathbf{K}\right) & E_{j}(\mathbf{r}) \exp \left(-\frac{i}{\hbar} \boldsymbol{\phi} \cdot \mathbf{K}\right) \\
\approx & E_{j}(\mathbf{r})+\frac{i}{\hbar} \phi_{i}\left[K_{i}, E_{j}(\mathbf{r})\right] \\
= & E_{j}(\mathbf{r})+\phi_{i} \int d^{3} r^{\prime}\left[r_{i}^{\prime} B_{p}\left(\mathbf{r}^{\prime}\right) \varepsilon_{p q j} \nabla_{q}^{\prime} \delta\left(\mathbf{r}-\mathbf{r}^{\prime}\right)\right. \\
\quad & \left.-t \varepsilon_{i l m} E_{j}\left(\mathbf{r}^{\prime}\right) \varepsilon_{m q j} \nabla_{q}^{\prime} \delta\left(\mathbf{r}-\mathbf{r}^{\prime}\right)\right] \\
= & E_{j}(\mathbf{r})+\phi_{i}\left[-\varepsilon_{i j p} B_{p}(\mathbf{r})+\left(r_{i} \frac{\partial}{\partial t}+t \nabla_{i}\right) E_{j}(\mathbf{r})\right],
\end{aligned}
$$

where we have made use of the first and fourth Maxwell equations. We can rewrite this in vector form as

$$
\begin{aligned}
\exp \left(\frac{i}{\hbar} \boldsymbol{\phi} \cdot \mathbf{K}\right) & \mathbf{E}(\mathbf{r}) \exp \left(-\frac{i}{\hbar} \boldsymbol{\phi} \cdot \mathbf{K}\right) \\
& \approx \mathbf{E}(\mathbf{r})+\boldsymbol{\phi} \cdot\left(\mathbf{r} \frac{\partial}{\partial t}+t \nabla_{i}\right) \mathbf{E}(\mathbf{r})-\boldsymbol{\phi} \times \mathbf{B}(\mathbf{r}),
\end{aligned}
$$

which we recognise as the infinitessimal Lorentz transformation (19).

We can proceed in a similar manner to obtain the transformation of the magnetic field. It is more elegant, however, to rely on the Heaviside-Larmor symmetry which requires that

$$
\begin{aligned}
\exp \left(\frac{i}{\hbar} \boldsymbol{\phi} \cdot \mathbf{K}\right)[ & \cos \theta \mathbf{E}+\sin \theta \mathbf{B}] \exp \left(-\frac{i}{\hbar} \boldsymbol{\phi} \cdot \mathbf{K}\right) \\
\approx \cos \theta & {\left[\mathbf{E}+\boldsymbol{\phi} \cdot\left(\mathbf{r} \frac{\partial}{\partial t}+t \nabla_{i}\right) \mathbf{E}-\boldsymbol{\phi} \times \mathbf{B}\right] } \\
& +\sin \theta\left[\mathbf{B}+\boldsymbol{\phi} \cdot\left(\mathbf{r} \frac{\partial}{\partial t}+t \nabla_{i}\right) \mathbf{B}+\boldsymbol{\phi} \times \mathbf{E}\right]
\end{aligned}
$$

which, for $\theta=\pi / 2$, gives the required Lorentz transformation of the magnetic field.

\section{References}

[1] Allen L, Barnett S M and Padgett M J 2003 Optical Angular Momentum (Bristol: Institute of Physics) 
[2] Allen L, Padgett M J and Babiker M 1999 Prog. Opt. 39291

[3] Yao A M and Padgett M J Adv. Opt. Photon. To be published

[4] Allen L, Beijersbergen M W, Spreeuw R J C and Woerdman J P 1992 Phys. Rev. A 458185

[5] He H, Friese M E J, Heckenberg N R and Rubinsztein-Dunlop 1991 Phys. Rev. Lett. 75826

[6] Simpson N B, Dholakia K, Allen L and Padgett M J 1997 Opt. Lett. 2252

[7] O'Neil A T, MacVicar I, Allen L and Padgett M J 2002 Phys. Rev. Lett. 88053601

[8] Mair A, Vaziri A, Weihs G and Zeilinger A 2001 Nature 412313

[9] Franke-Arnold S, Barnett S M, Padgett M J and Allen L 2002 Phys. Rev. A 65033823

[10] Leach J, Jack B, Romero J, Jha A K, Yao A M, Franke-Arnold S, Ireland D G, Boyd R W, Barnett S M and Padgett M J 2010 Science 329662

[11] Götte J B, Franke-Arnold S and Barnett S M 2006 J. Mod. Opt. 53627

[12] Barnett S M and Allen L 1994 Opt. Commun. 110670

[13] Barnett S M 2002 J. Opt. B: Quantum Semiclass. Opt. 4 S1

[14] Bliokh K Y, Alonso M A, Ostrovskaya E A and Aiello A (To be published)

[15] van Enk S and Nienhuis G 1994 Europhys. Lett. 25497

[16] van Enk S and Nienhuis G 1994 J. Mod. Opt. 41963

[17] Barnett S M 2010 J. Mod. Opt 571339

[18] Weinberg S 1972 Gravitation and Cosmology: Principles and Applications of the General Theory of Relativity (New York: Wiley)

[19] Feynman R P, Leighton R B and Sands M 1963 The Feynman Lectures in Physics Vol. I (Reading Massachusetts: Addison Wesley)

[20] Feynman R P, Leighton R B and Sands M 1963 The Feynman Lectures in Physics Vol. II (Reading Massachusetts: Addison Wesley)

[21] Jackson J D 1999 Classical Electrodynamics 3rd edn (New York: Wiley)

[22] Stephenson G and Radmore P M 1990 Advanced Mathematical Methods for Engineering and Science Students (Cambridge: Cambridge University Press)

[23] Møller C 1952 The Theory of Relativity (Oxford: Oxford University Press)

[24] Jones H F 1998 Groups, Representations and Physics 2nd edn (Bristol: Institute of Physics Publishing)

[25] Weber H 1901 Die Partellen Differential-Gleichung der Mathematischen Physik nach Riemann's Vorlesungen (Braunschweig: Friedrich Vieweg und Sohn)

[26] Silberstein L 1914 The Theory of Relativity (London: MacMillan)

[27] Białynicki-Birula I 1996 Progress in Optics 36245

[28] Bateman H 1915 The Mathematical Analysis of Electrical and Optical Wave-Motion (Cambridge: Cambridge University Press) Reprinted 1955 (New York: Dover)

[29] Stratton J A 1941 Electromagnetic Theory (New York: McGraw-Hill)

[30] Heaviside O 1892 Phil. Trans. R. Soc. Lond. A 183423

[31] Larmor J 1897 Phil. Trans. R. Soc. Lond. A 190205

[32] Rose M E 1955 Multipole Fields (New York: Wiley)

[33] Berry M V 2009 J. Opt. A: Pure Appl. Opt. 11094001

[34] Cohen-Tannoudji C, Dupont-Roc J and Grynberg G 1989 Photons and Atoms (New York: Wiley)

[35] Barnett S M and Zambrini R 2005 in Quantum Imaging Kolobov M I ed. (New York: Springer) 